\title{
Early outcomes of transatrial mitral valve replacement in severe mitral annular calcification
}

\author{
Yuji Kawano, MD, ${ }^{\mathrm{a}, \mathrm{b}}$ Paige Newell, MD, ${ }^{\mathrm{a}}$ Morgan Harloff, MD, ${ }^{\mathrm{a}}$ Sameer Hirji, MD, ${ }^{\mathrm{a}}$ Edward Percy, MD, ${ }^{\mathrm{c}}$ \\ Pinak Shah, MD, ${ }^{d}$ and Tsuyoshi Kaneko, MD ${ }^{\mathrm{a}}$
}

\section{ABSTRACT}

Objective: Mitral valve replacement (MVR) in the setting of severe mitral annular calcification is a technically challenging operation with increased morbidity and mortality. Transseptal/apical transcatheter MVR (TMVR) in mitral annular calcification has emerged as an option for these cases, although may not be feasible due to anatomical reasons. Transatrial TMVR is a potential treatment option for this subgroup of patients.

Methods: Patients who underwent transatrial TMVR between June 2018 and November 2020 at a single institution were included. Patients were selected by a structural heart team based on their surgical risk, pattern of mitral annular calcification, risk of valve migration, left ventricular outflow obstruction, and paravalvular leak.

Results: A total of 11 patients underwent transatrial TMVR. Mean patient age was 74.2 years and mean Society of Thoracic Surgeons predicted risk of mortality score was $9.1 \%$. All patients had the presence of both mitral stenosis and regurgitationdominant etiology - was mitral stenosis in $81.2 \%$, and mitral regurgitation in $18.8 \%$. Among patients, $54.5 \%$ had a concomitant cardiac procedure. There was no in-hospital or 30-day mortality. Technical success defined by the Mitral Valve Academic Research Consortium was achieved in $90.9 \%$ of patients. Postoperative paravalvular leak was mild or less in all patients.

Conclusions: In this series, transatrial TMVR was shown to be a safe and effective treatment option for patients who are high risk for surgical MVR and should be in surgeons' armamentarium in the treatment of this high-risk patient population. Dissemination of safe technique will be critical in the successful conduct of this surgery. (JTCVS Techniques 2021;9:49-56)

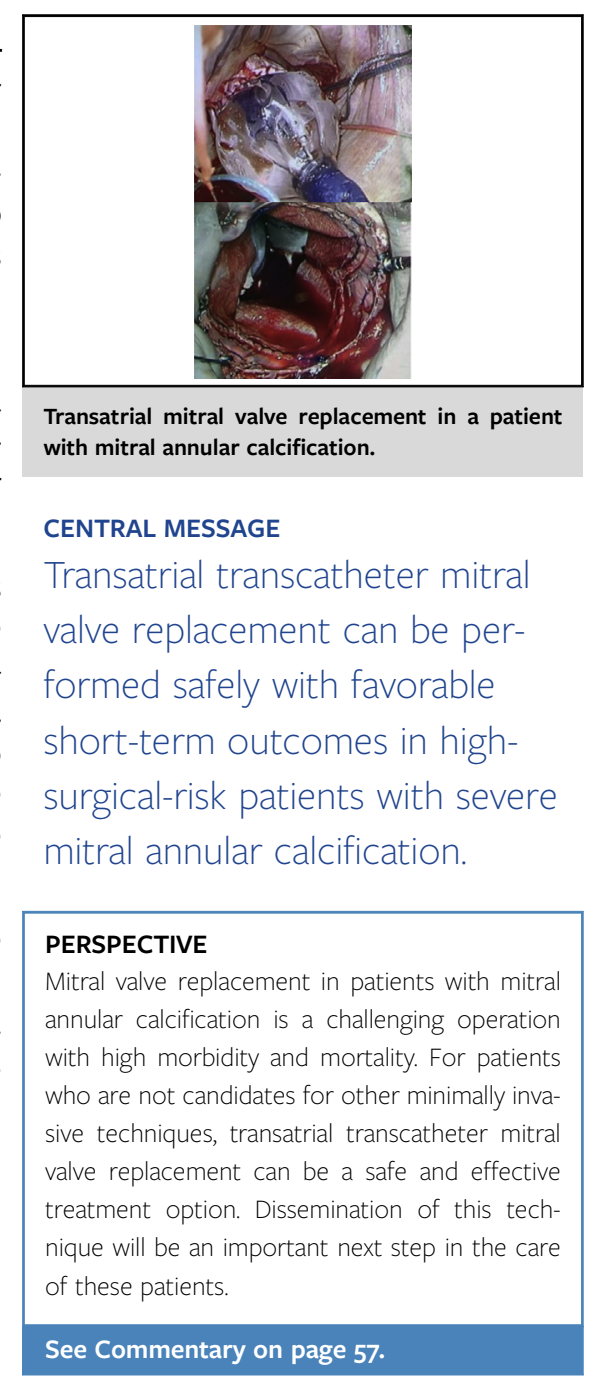

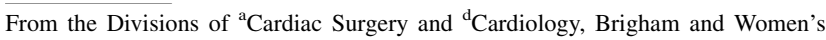
Hospital, Harvard Medical School, Boston, Mass; 'bivision of Cardiac Surgery, Emory University School of Medicine, Atlanta, Ga; and ${ }^{\mathrm{c}}$ Division of Cardiac Surgery, University of British Columbia, Vancouver, British Columbia, Canada.

Drs Kawano and Newell contributed equally to this article.

Received for publication Jan 18, 2021; accepted for publication June 7, 2021; available ahead of print June 17, 2021.

Address for reprints: Tsuyoshi Kaneko, MD, Division of Cardiac Surgery, Brigham and Women's Hospital, 15 Francis St, Boston, MA 02115 (E-mail: tkaneko2@ partners.org)

2666-2507

Copyright (C) 2021 The Author(s). Published by Elsevier Inc. on behalf of The American Association for Thoracic Surgery. This is an open access article under the CC BY-NC-ND license (http://creativecommons.org/licenses/by-nc-nd/4.0/).

https://doi.org/10.1016/j.xjtc.2021.06.015
}

$\checkmark$ Video clip is available online.

Mitral valve replacement (MVR) in the setting of severe mitral annular calcification (MAC) is among the most challenging surgeries faced by cardiac surgeons. The presence of extensive calcium deposits in the mitral annulus complicates the surgery by potential injury to surrounding structures during debridement, impediment of suture 


\section{Abbreviations and Acronyms \\ $\mathrm{CT}=$ computed tomography \\ EOA = effective orifice area \\ LVOT $=$ left ventricular outflow tract \\ MAC = mitral annular calcification \\ MVR = mitral valve replacement \\ PVL = paravalvular leak \\ TAVR $=$ transcatheter aortic valve replacement \\ $\mathrm{THV}=$ transcatheter heart valve \\ $\mathrm{TMVR}=$ transcatheter mitral valve replacement}

placement through the calcium, and a suboptimal size of prosthesis placed inside the MAC. To treat these problems, various surgical strategies, including segmental decalcification, ${ }^{1}$ en bloc decalcification along with suture or patch reconstruction of the annulus, ${ }^{2,3}$ atrial sliding plasty, and anterior leaflet transposition ${ }^{4}$ have been previously described. These techniques are effective and feasible, but they necessitate extended cardiopulmonary bypass and aortic crossclamp times, which adversely affect clinical outcomes. Moreover, these maneuvers may be associated with increased risk for devastating complications, including atrioventricular dissociation, injury to the circumflex coronary artery, and disturbances of the conduction system.

Transcatheter aortic valve replacement (TAVR) was approved by the Food and Drug Administration for lowsurgical-risk patients with severe aortic stenosis in August 2019. ${ }^{5}$ Balloon-expandable TAVR valves including the Sapien 3 Balloon-Expandable Transcatheter Heart Valve System (Edwards Lifesciences, Irvine, Calif), adhere to the calcium inside the aortic annulus for stability. Using this concept, transcatheter MVR (TMVR) has been introduced as a new treatment option to address mitral valve disease with MAC. ${ }^{6-8}$ The primary access route for TMVR in MAC in the early series has been transapical or transseptal. However, not all patients are eligible for these access routes for various reasons, including unfavorable distribution of MAC, high risk for left ventricular outflow tract (LVOT) obstruction, and need for concomitant surgical interventions. Transatrial TMVR with the Sapien 3 valve has emerged as an alternative solution in these difficult situations. ${ }^{9-14}$ Unlike the other percutaneous transcatheter approaches, this technique carries the risks of an open procedure, but allows the surgeon to size the valve, deploy the prosthesis under direct visualization, prevent valve migration by placing anchoring sutures, reduce paravalvular leak (PVL) with a felt-reinforced valve skirt, and mitigate potential LVOT obstruction by excising the anterior mitral leaflet.

The aim of this study is to show that, with careful patient selection, these unique features of transatrial TMVR contribute to improved clinical outcomes in patients at high surgical risk who are not eligible for other, lessinvasive approaches. We present the results of our singlecenter experience in 11 consecutive transatrial TMVR cases in patients with severe MAC.

\section{METHODS}

Between June 2018 and November 2020, patients referred for the treatment of MAC associated with symptomatic severe mitral stenosis, regurgitation, or mixed mitral valve disease were assessed by the structural heart team and evaluated for TMVR via transseptal, transapical, or transatrial approaches. We had a total of 104 mitral consults in our structural heart clinic. Of those, 18 were candidates for TMVR and were screened for a transatrial approach due to MAC: 8 patients underwent transatrial TMVR, 5 underwent transseptal TMVR, and the remaining 5 opted out of a TMVR approach or are still pending workup. The remaining 3 patients in our case series came from direct admission to the hospital or an outside hospital transfer. Patients were assessed for their candidacy for the transatrial approach primarily based on their ability to withstand open cardiac surgery. Patients with any severe organ failure, except end-stage renal disease, were not eligible for the transatrial approach. Patients who were determined to be frail, as defined as a score of 3 to 5 on the Johns Hopkins Frailty Assessment, ${ }^{15}$ were also not eligible for transatrial TMVR. Circumferential MAC did not preclude patients from the transatrial approach in the case that a patient was a surgical candidate. Patients who were not surgical candidates with circumferential MAC and low-risk of LVOT obstruction were preferentially treated with the transseptal approach: we performed 5 transseptal TMVRs during this time period. All 11 patients who underwent transatrial TMVR during this period were included in this study. Emergency or salvage cases were excluded. In selective cases, preoperative cardiac computed tomography (CT) scans were obtained to analyze the distribution and burden of MAC as well as the mitral annular area. Valve areas up to $1000 \mathrm{~mm}^{2}$ were considered. In addition, predicted neo-LVOT area was calculated with a simulated transcatheter heart valve in accordance with commercially available imaging software (Vitrea; Vital Images, Minnetonka, Minn) in selected patients. The pattern of MAC and additional criteria, including high risk of valve migration, LVOT obstruction, and PVL, were considered before patient selection. All cases were performed by a single cardiac surgeon at a single institution. The final decision to proceed with transatrial TMVR was made after the mitral valve was directly visualized during surgery. This study was approved by the institutional review board (No. 2010P000292; December 11, 2019) and complied with all national and institutional regulations for human subjects research.

\section{Surgical Technique}

The mitral valve was approached via median sternotomy or minimally invasive anterior right thoracotomy depending on patient body habitus and need for concomitant surgery. We cannulated centrally for median sternotomy or peripherally through the right femoral artery and vein for the right thoracotomy approach. After initiation of cardiopulmonary bypass, the ascending aorta was crossclamped and cardiac arrest was achieved. The mitral valve was exposed via left atriotomy through Sondergaard's groove. The anterior leaflet was resected along the annulus (Figure 1, A). The subvalvular tissue, chordae, and papillary muscles were resected when necessary to accommodate the fully expanded valve. Pledgeted 2-0 Ethibond (Johnson \& Johnson, Franklin Lakes, NJ) annular sutures were placed in noneverting mattress fashion. At least 3 annular sutures were placed, 1 at each trigone and 1 in the middle of the posterior annulus, even when the annulus was extensively calcified (Figure $1, B$ ). In patients with a large valve area, the rim of the anterior leaflet was left intact and circumferential sutures were placed. For an annulus that had incomplete MAC, the locations with no MAC were reinforced with these annular sutures. These sutures served to anchor the prosthesis in place. Minimal attempts were 


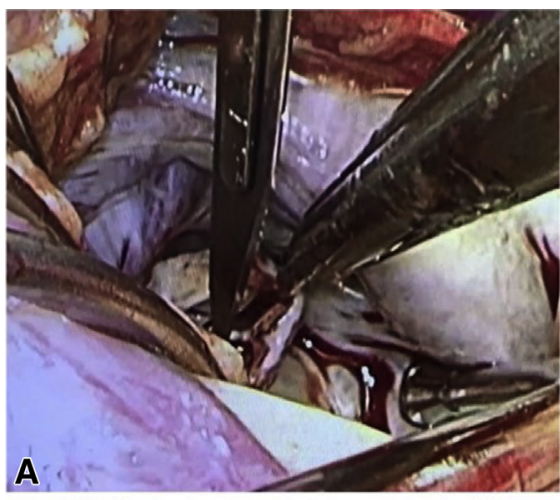

Anterior leaflet is resected to reduce the risk of LVOT obstruction

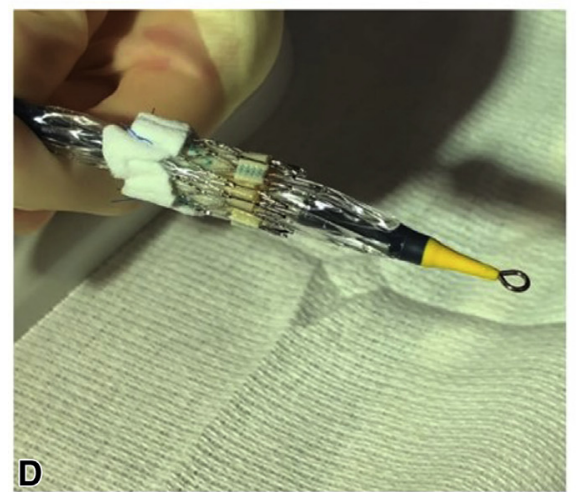

Valve is crimped and loaded on the delivery system (skirt towards handle)

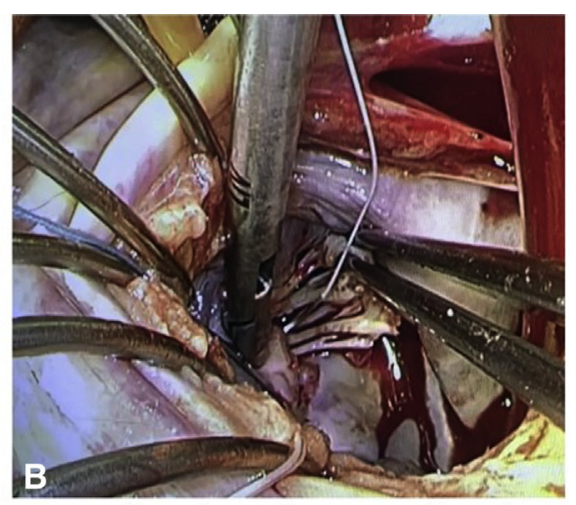

Annular sutures are placed, avoiding area with MAC when able

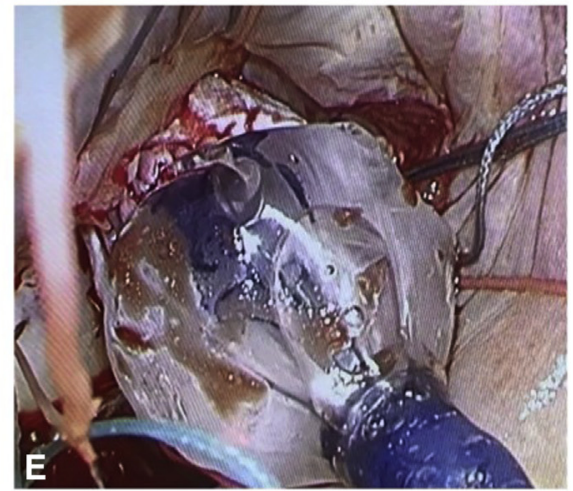

Valve is deployed under direct visualization

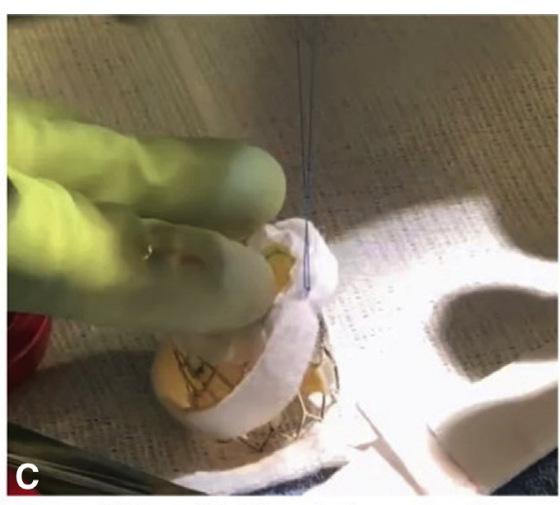

Valve skirt is reinforced with a Teflon felt strip

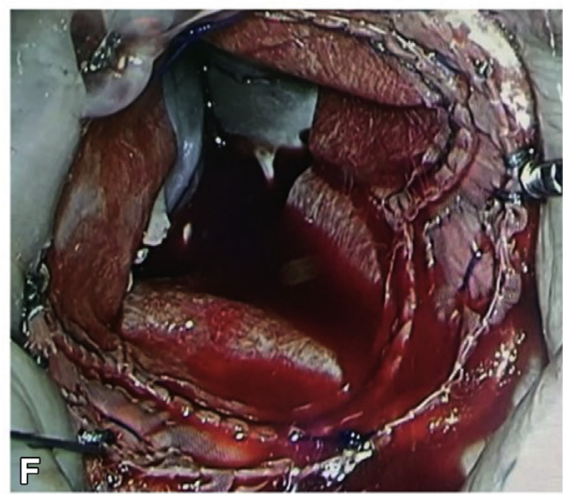

Annular sutures are tied down to secure the valve

FIGURE 1. Intraoperative images of transatrial mitral valve replacement (TMVR). For high- or prohibitive-surgical risk patients with mitral annular calcification $(M A C)$, a transatrial TMVR can be a safe and effective treatment option. This figure shows 6 distinct intraoperative steps for the successful execution of this procedure. After exposing the mitral valve, the anterior leaflet of the valve is excised to reduce the risk of left ventricular outflow tract (LVOT) obstruction (A). Next, annular sutures are placed, avoiding MAC when possible (B). Next the Sapien 3 valve (Edwards Lifesciences, Irvine, Calif) is prepped on the back table, first by reinforcing the valve skirt with a polytetrafluoroethylene felt strip (C) and then loading the valve on the delivery system with the skirt toward the handle (D). Finally, under direct visualization, the surgeon deploys the valve (E) and secures it in place by tying down the previously placed annular sutures (F). This technique allows the surgeon to address LVOT obstruction, paravalvular leak, and concomitant procedures at the time of MVR for these highrisk patients.

made to debride the calcium to avoid any potential injury to surrounding structures such as the circumflex coronary artery or conduction system.

The Sapien 3 valve was prepared on a back table. The size of the valve was measured on preoperative CT scan if available and confirmed with balloon sizing under direct visualization before deployment. After the valve was opened, a felt strip was wrapped around the valve cuff and sewn with 4-0 Prolene mattress sutures to reinforce the sealing ability against the calcified annulus (Figure 1,C).

The valve was crimped and loaded onto the balloon delivery system (Certitude; Edwards Lifesciences) on the back table after confirmation of valve size (Figure 1,D). It is important to note that the valve is mounted on the delivery system with the skirt toward the handle, opposite the direction in which it would be loaded for TAVR. A standard $J$-wire was inserted into the left ventricle under direct visualization. The use of a soft guidewire was necessary to avoid injury to the chordae, papillary muscles, or left ventricular apex. The delivery system was advanced over the wire and brought into the mitral annulus where the sealing cuff was aligned with the mitral annular plane. The valve was deployed under slow inflation with direct visualization (Figure 1,E). After deployment of the valve, the previously placed $2-0$ Ethibond sutures were placed through the valve cuff and tied down (Figure 1, F). In cases where TAVR had previously been performed, caution was taken to position the valves in such a way that the stents of the 2 valves would not interfere with each other.

The left ventricle was filled with normal saline and pressurized to interrogate the stability of the valve and ensure the absence of PVL. Extra annular sutures were added through the atrial tissue if PVL was detected. The left atrium was closed with 3-0 Prolene double running sutures. The patient was then separated from cardiopulmonary bypass. At this point, transesophageal echocardiogram was performed to confirm satisfactory valve positioning, measure valve gradients, and verify the absence of PVL and LVOT obstruction. After standard de-airing maneuvers, all cannulas were removed. Hemostasis was achieved, and the chest was closed in the standard fashion. Intraoperative images are presented in Figure 1, $A$ through $F$, and the step-by-step sequence for delivering a Sapien 3 valve in Figure 2. A video summary of the intraoperative steps is presented in Video 1.

All patients were transferred to the cardiac intensive care unit postoperatively. All were successfully discharged either home or to a rehabilitation facility after recovery. Lifelong aspirin 81 milligrams and a minimum of 3 months of warfarin with a target international normalized ratio of 2.0 to 3.0 were continued unless bleeding complications were observed. An 


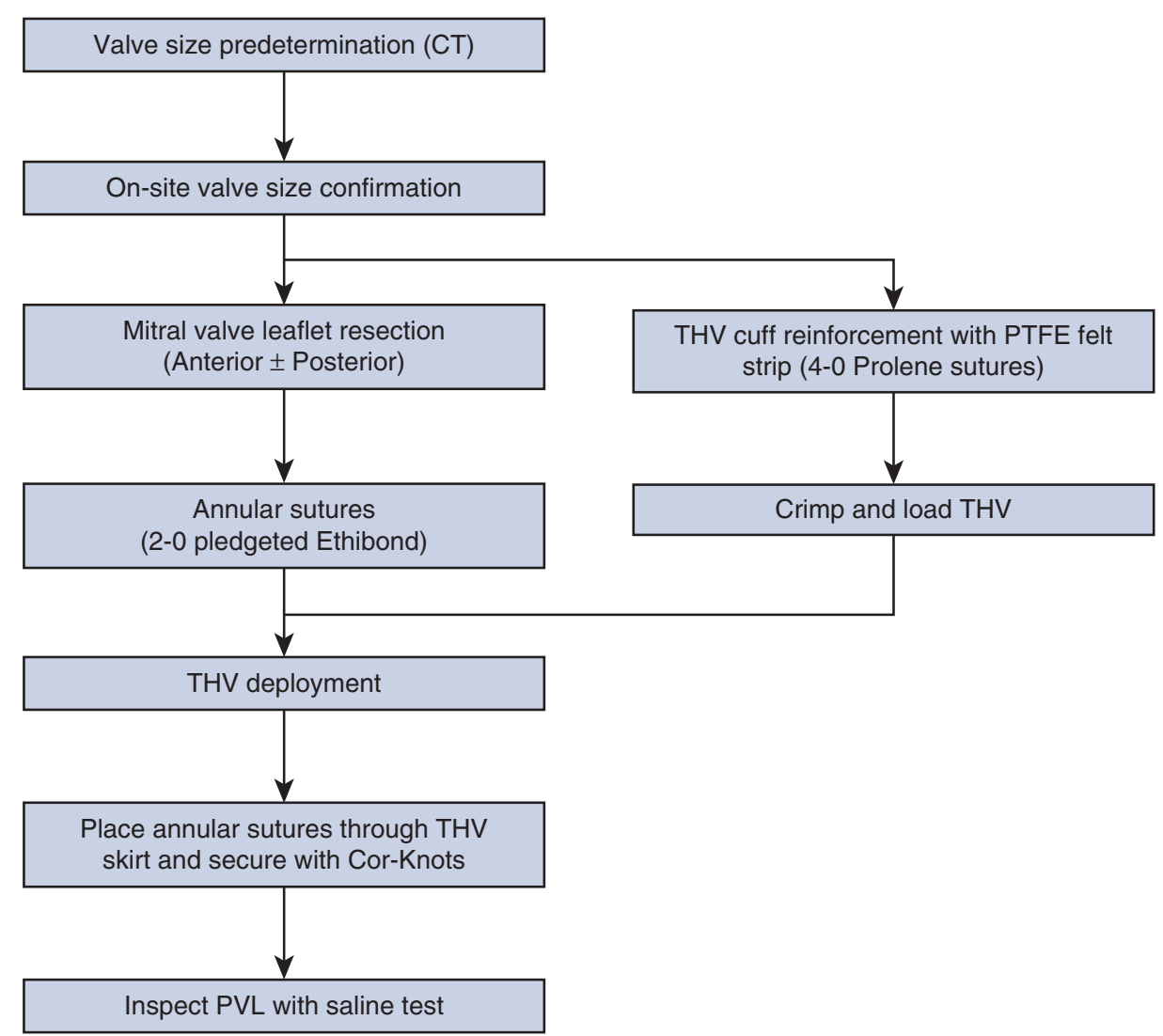

$\mathrm{CT}=$ computed tomography; THV = transcatheter heart valve; PTFE = polytetrafluoroethylene

FIGURE 2. Step-by-step sequence for deploying Sapien 3 valve (Edwards Lifesciences, Irvine, Calif). Shown is the step-by-step sequence for deploying a Sapien 3 valve during transatrial transcatheter mitral valve replacement. The valve size will be predetermined based on preoperative computed tomography (CT). Once intraoperative, the valve size should be confirmed. Either simultaneously or in succession, the mitral valve leaflet can be resected and 2-0 pledgeted Ethibond (Johnson \& Johnson, Franklin Lakes, NJ) sutures can be placed around the annulus. At the same time on the back table, the transcatheter heart valve $(T H V)$ should be reinforced with a polytetrafluoroethylene ( $P T F E$ ) felt strip using 4-0 Prolene sutures. The THV can then be crimped and loaded onto the delivery system. Now that the annular sutures are in place and the THV is prepared, the THV should be deployed and the annular sutures should be secured through the THV skirt and secured with Cor-Knots (Litigation Solutions Inc, Greenwood Village, Colo). Once all of the sutures have been secured, the surgeon can inspect for paravalvular leak $(P V L)$ using a saline test.

echocardiogram was typically performed within 30 days of surgery. Further clinical and echocardiographic follow-up was scheduled 1 year after surgery and yearly thereafter.

\section{RESULTS}

A total of 11 patients were included in this study. Mean patient age was 74.3 years, $81.8 \%$ were women, and mean Society of Thoracic Surgeons predicted risk of mortality score was $9.06 \%$. Among patients, $54.5 \%$ had prior cardiac surgery, and $9.1 \%$ had previous mitral valve procedures. The majority of patients had multiple reasons for being candidates for a transatrial approach with $81.8 \%$ of patients having an incomplete MAC pattern, $45.5 \%$ of patients needing concomitant cardiac surgery, and $81.8 \%$ of patients being at high-risk of LVOT obstruction. Preoperatively, 10 patients had a dedicated gated cardiac CT. Median predicted neo-LVOT was $1.15 \mathrm{~cm}^{2}$ (interquartile range,
0.81-1.6 $\mathrm{cm}^{2}$ ) and 9 patients had high-risk for LVOT obstruction (defined as $<1.5 \mathrm{~cm}^{2}$ ). ${ }^{16}$ Nine patients had incomplete MAC in the anterior annulus. Patient baseline characteristics are listed in Table 1.

A hybrid operating room was utilized for the first case; however, radiograph visualization of the MAC was not more accurate than direct visualization of the valve and subsequent cases were performed in standard operating rooms. Two patients underwent surgery via right thoracotomy and 9 patients underwent full median sternotomy. Patients who underwent isolated transatrial TMVR or concomitant tricuspid valve procedures were selected for the right thoracotomy approach, otherwise median sternotomy was used to accommodate the other concomitant procedures. All reperative cases were performed via redo sternotomy based on the surgeon's experience. Five patients required concomitant cardiac surgery: 2 patients required septal myectomy, 1 


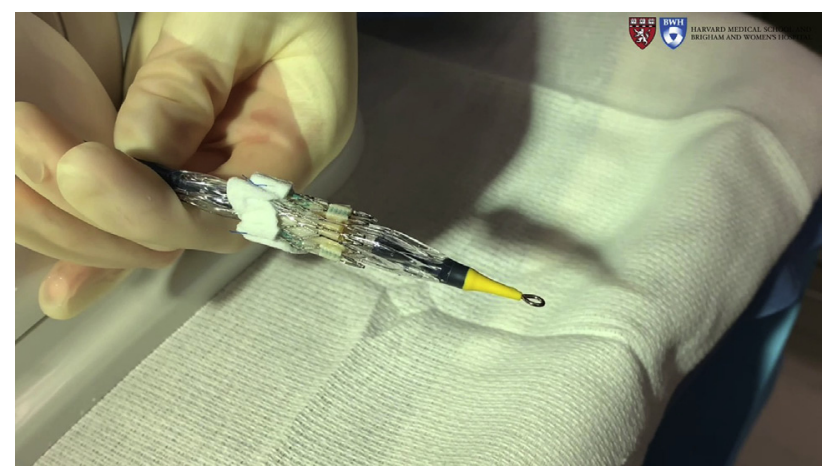

VIDEO 1. Transatrial transcatheter mitral valve replacement (TMVR) intraoperative video. Shown is an operative video of the step-by-step sequence of transatrial TMVR performed in this present study. The video includes subtitles explaining each key step in this particular procedure. Video available at: https://www.jtcvs.org/article/S2666-2507(21)00411-9/ fulltext.

required coronary artery bypass grafting, and 2 required aortic valve replacement and tricuspid repair. On direct visualization of the mitral valve intraoperatively, significant MAC was located along the posterior annulus extending to the anterior annulus in all patients, with 2 patients having circumferential MAC. All 11 patients had the anterior leaflet resected, regardless of predicted neo-LVOT. After directly sizing the valve intraoperatively, the size of the implanted transcatheter heart valve (THV) was $29 \mathrm{~mm}$ in 7 patients and $26 \mathrm{~mm}$ in 4 patients. Technical success, defined by the Mitral Valve Academic Research Consortium, ${ }^{17}$ was achieved in 10 out of 11 patients. The patient who did not have technical success required a second valve-invalve THV to be deployed during the index operation. This was due to the cuff being ventricular in the anterior annulus secondary to poor visualization from the patient's previous TAVR. Operative details are shown in Table 2.

Postoperatively, there were no in-hospital or 30-day mortalities. A permanent pacemaker implantation was required in $18.2 \%$ of patients for complete heart block. One patient required an urgent reoperation on postoperative day 2 due to LVOT obstruction and hemolytic anemia, which was successfully treated by explanting the THV and performing a surgical MVR with a $25 \mathrm{~mm}$ bioprosthetic valve. This same patient had a complicated postoperative course and experienced a stroke and had a new hemodialysis requirement. This patient survived, but was subsequently rehospitalized for heart failure exacerbation. Full postoperative outcomes are summarized in Table 3.

All patients received an immediate postprocedure echocardiogram, which showed none or trace PVL in 8 patients and mild PVL in 3 patients. Mean gradient through the THV was $<5 \mathrm{~mm} \mathrm{Hg}$ in 9 patients $(81.8 \%)$ and $5 \mathrm{~mm} \mathrm{Hg}$ in 2
TABLE 1. Baseline characteristics of patients undergoing transatrial transcatheter mitral valve replacement (TMVR) in mitral annular calcification (MAC) $(\mathbf{N}=\mathbf{1 1})$

\begin{tabular}{lc}
\hline \multicolumn{1}{c}{ Characteristic } & Result \\
\hline Age (y) & $74.3 \pm 9.0$ \\
Female sex & $9(81.8)$ \\
\hline BMI & $34.2 \pm 10.5$ \\
\hline DM & $6(54.5)$ \\
\hline CKD & $4(36.4)$ \\
HTN & $10(90.9)$ \\
\hline HLD & $5(45.5)$ \\
PAD & $1(9.1)$ \\
\hline Previous TIA or CVA & $3(27.3)$ \\
CAD & $2(18.1)$ \\
\hline Previous MI & $1(9.1)$ \\
Prior PCI & $0(0)$ \\
\hline Afib & $4(36.4)$ \\
COPD & $3(27.3)$ \\
\hline Baseline NYHA functional class & $2.5 \pm 0.52$ \\
Ejection fraction & $64.4 \pm 7.9$ \\
Prior TAVR & $4(36.4)$ \\
\hline Prior mitral valve procedure & $1(9.1)$ \\
\hline Severe MR & $1(9.1)$ \\
\hline Severe MS & $9(81.8)$ \\
\hline STS PROM MV gradient (mm Hg) & $9.1 \pm 6.5$ \\
Reason for transatrial approach & $9(81.8)$ \\
\hline Concomitant cardiac surgery & $9(81.8)$ \\
\hline
\end{tabular}

Values are presented as mean \pm standard deviation or $\mathrm{n}(\%) . B M I$, Body mass index; $D M$, diabetes mellitus; $C K D$, chronic kidney disease; $H T N$, hypertension; $H L D$, hyperlipidemia; $P A D$, peripheral arterial disease; TIA, transient ischemic attack; $C V A$, cerebrovascular accident; $C A D$, coronary artery disease; $M I$, myocardial infarction; $P C I$, percutaneous coronary intervention; $A f i b$, atrial fibrillation; $C O P D$, chronic obstructive pulmonary disease; NYHA, New York Heart Association; TAVR, transcatheter aortic valve replacement; $M R$, mitral valve regurgitation; MS, mitral valve stenosis; $M V$, mitral valve; STS PROM, Society of Thoracic Surgeons predicted risk of mortality Score; LVOT, left ventricular outflow tract.

patients $(18.1 \%)$. LVOT obstruction, defined by peak gradient $>30 \mathrm{~mm} \mathrm{Hg}$, was observed in 2 patients $(18.1 \%)$. Among these, 1 had undergone previous TAVR with a self-expandable THV (Evolut Pro; Medtronic, Minneapolis, Minn) whereas 1 other patient previously underwent TAVR with a Sapien 3 and had baseline LVOT obstruction at the time of preoperative assessment. For patients who received a 30-day echocardiogram after surgery, PVL was none or trace. Echocardiographic findings are summarized in Table 3. 
TABLE 2. Operative characteristics of patients undergoing transatrial transcatheter mitral valve replacement (TMVR) in mitral annular calcification (MAC) $(\mathrm{N}=\mathbf{1 1})$

\begin{tabular}{lc}
\hline \multicolumn{1}{c}{ Characteristic } & Result \\
\hline Approach & $9(81.8)$ \\
$\quad$ Full sternotomy & $2(18.1)$ \\
Right thoracotomy & $1(9.1)$ \\
Concomitant CABG & $2(18.2)$ \\
Concomitant septal myectomy & $2(18.2)$ \\
Concomitant AVR & $119.8 \pm 44.7$ \\
CPB Time (min) & $86.2 \pm 34.6$ \\
Crossclamp time (min) & $7(63.6)$ \\
THV* & $4(36.4)$ \\
Sapien 329 mm & $10(90.9)$ \\
\hline Sapien 326 mm & \\
Technical success defined by MVARC
\end{tabular}

Values are presented as mean \pm standard deviation or $\mathrm{n}(\%) . C A B G$, Coronary artery bypass graft; $A V R$, aortic valve replacement; $C P B$, cardiopulmonary bypass; $T H V$, transcatheter heart valve; $M V A R C$, mitral valve academic research consortium. *Manufactured by Edwards Lifesciences, Irvine, Calif.

\section{DISCUSSION}

Transatrial TMVR has been described in the literature, but the present study demonstrates that despite patients having high surgical risk profiles, this technique can be performed safely and with excellent outcomes as demonstrated by no significant postoperative PVL and no in-hospital or 30-day mortality in our patient cohort. The lack of mortality is particularly important with the transatrial approach being an open surgery, which increases the inherent risk of the procedure but provides the surgeon with an opportunity to address LVOT obstruction and concomitant surgery in the same operation. Less-invasive approaches are ideal for high-risk patients, but we believe transatrial TMVR bridges the gap between conventional mitral valve surgery and transcatheter procedures and serves a unique patient population that is best served by this hybrid technique without incurring a substantial increased mortality risk. Transatrial TMVR is a safe and effective treatment option for patients who are at high-risk for surgical MVR and should be in the surgeon's armamentarium in the treatment of this high-risk patient population. Dissemination of safe technique will be critical in the successful conduct of this surgery.

Thirty-day and in-hospital mortality for all TMVR cases in MAC has been reported as high as $25 \%$ in previous studies. $^{8}$ Of the possible approaches, transapical is the most common approach at more than $40 \%$ of cases, followed by transseptal at more than $30 \%$, and lastly transatrial at just under $20 \%{ }^{18}$ The transseptal approach has the advantage of being a minimally invasive percutaneous procedure, and a transapical approach can avoid cardiopulmonary bypass, whereas the transatrial approach is only possible if the patient is a surgical candidate. The original
TABLE 3. Echocardiographic and postprocedural outcomes for patients undergoing transatrial transcatheter mitral valve replacement (TMVR) in mitral annular calcification (MAC) $(\mathbf{N}=11)$

\begin{tabular}{ll}
\multicolumn{1}{c}{ Outcome } & Result \\
\hline 30-day outcomes & \\
In-hospital mortality & $0(0)$ \\
30-day mortality & $0(0)$ \\
Stroke & $1(9.1)$ \\
Cardiac surgery reoperation & $1(9.1)$ \\
Hemolytic anemia & $1(9.1)$ \\
Vascular access complication & $1(9.1)$ \\
Arrhythmia & $7(63.6)$ \\
Permanent pacemaker implantation & $2(18.2)$ \\
New hemodialysis requirement & $1(9.1)$ \\
Blood transfusion & $3(27.3)$ \\
ICU LOS (d) & $10.6 \pm 20.6$ \\
Hospital LOS (d) & $19.1 \pm 20.2$ \\
Postprocedure echocardiographic outcomes & \\
Postoperative PVL & \\
None or trace & $8(72.7)$ \\
Mild & $3(27.3)$ \\
Moderate or severe & $0(0)$ \\
Mean THV gradient $<5 \mathrm{~mm} \mathrm{Hg}$ & $9(81.2)$ \\
LVOT gradient $\geqq 30 ~ m m ~ H g$ & $2(18.2)$ \\
\hline
\end{tabular}

Values are presented as mean \pm standard deviation or $\mathrm{n}(\%)$. ICU, Intensive care unit; $L O S$, length of stay; $P V L$, paravalvular leak; $T H V$, transcatheter heart valve; $L V O T$, left ventricular outflow tract.

study examining the transatrial TMVR approach in patients with MAC had an in-hospital mortality of $19 \%$ and 30-day mortality of $27 \% .^{9}$ In contrast, more recent studies have demonstrated improvement in outcomes with 30-day mortality rates of $12.5 \%{ }^{19}$ and $0 \% .{ }^{10}$ The $0 \%$ in-hospital and 30-day mortality in this study further supports that transatrial TMVR is a safe and effective treatment option for qualifying patients. We believe that with careful patient selection and thorough preoperative workup, the true perioperative mortality for transatrial TMVR is likely lower than previously reported.

Patients with MAC undergoing MVR have been shown to be independently associated with worse postoperative outcomes, including reoperation, postoperative morbidity, and operative mortality ${ }^{20}$ than patients without MAC. MAC presents the cardiac surgeon with complex intraoperative decision making because there currently is no universally accepted surgical approach. Options for dealing with MAC during mitral valve surgery evolves around making the decision to either resect or respect. Dense calcium deposits around the mitral annulus can be resected by cutting into the endocardium, followed by annular reconstruction. Removal of calcium allows for the implantation of a larger prosthetic valve with better sealing against the annulus due to better tissue compliance. However, this increases the complexity of the surgery, the risk for atrioventricular 
groove dissociation, and potential injury to the circumflex coronary artery. Alternatively, respecting MAC by leaving calcium behind presents a challenge because placing sutures through or beyond the calcium is technically demanding and carries the risk of injuring surrounding structures. The transatrial TMVR technique described herein minimizes these risks because it does not require debridement or circumferential annular sutures through MAC, but still allows for implantation of a balloon-expandable THV. Specifically, minimizing any debridement and reinforcing the valve skirt with a felt strip we found to adequately achieve our desired postoperative outcomes. We also did not place sutures circumferentially; the sutures are placed in 3 locations (trigones and middle of P2 annulus) for all cases to avoid migration and additional sutures where the MAC is absent. We believe this shortens the crossclamp and cardiopulmonary bypass times. Some MAC patterns, such as MAC that protrudes into the leaflets, can make this technique more challenging because it does require debridement of the calcium. In these cases, we have found mild PVL postprocedurally and the surgeon should have a high index of suspicion and carefully evaluate the seating and seal of the valve.

One potential benefit of THV is the larger effective orifice area (EOA) compared with the same size surgical bioprosthesis. Data regarding in vivo EOA for the Sapien 3 valve compared with regular biologic valves for surgical MVR have consistently demonstrated a larger EOA in the same size Sapien 3 valve (EOA for $29 \mathrm{~mm}$ Sapien 3 being $2.7 \mathrm{~cm}^{2}$ compared with 1.9 to $2.0 \mathrm{~cm}^{2}$ for a $29-\mathrm{mm}$ bioprosthetic valve). ${ }^{21,22}$ These data support the potential advantage of utilizing THV for mitral valve disease with MAC. In our study, the mean transmitral gradient at 30days ranged from 5 to $8 \mathrm{~mm} \mathrm{Hg}$ except for the patient requiring the $25-\mathrm{mm}$ bioprosthetic valve who had a gradient of $12 \mathrm{~mm} \mathrm{Hg}$. This relatively high gradient might be explained by the existence of heavy calcium surrounding the THV, which might have hindered the full expansion of the valve. Furthermore, given the fact that the mitral annulus is $D$-shaped unlike the circular aortic valve annulus, the THVs might not have completely expanded to their full diameters due to limitations of the short radius on the annular plane. This incomplete expansion also has the potential to worsen prosthetic PVL, which is already a great concern in cases of severe MAC with early studies demonstrating $32.9 \%$ of cases having postprocedural mild PVL and $5 \%$ having grade 3 or higher PVL. ${ }^{8}$ Previous surgeons have proposed placing complete circumferential annular sutures during transatrial TMVR as a way to reduce PVL. ${ }^{10}$ However, we minimized the number of annular sutures to simplify the procedure and all patients had mild or less PVL. We believe this reduced rate of PVL can be attributed to the felt-reinforced valve skirt that facilitates a better seal against the calcified annulus, and that investigation of potential PVL with a pressurized left ventricle after valve deployment can provide the opportunity to add additional sutures should significant PVL be present. In this way, a felt skirt reinforced THV has the opportunity to provide a larger EOA and reduced PVL compared with biologic valves in patients with severe MAC.

Another potential complication after TMVR is LVOT obstruction, which has a reported in-hospital mortality rate exceeding $60 \% .{ }^{23}$ One option for addressing this and remaining endovascular is to perform laceration of the anterior mitral valve leaflet to prevent outflow obstruction before transseptal TMVR. ${ }^{23}$ Although this procedure has the benefit of being entirely transcatheter, it has mixed outcomes particularly for valve-in-MAC patients with a reported $13 \%$ 30-day mortality, $27 \%$ requiring additional procedures, and $23 \%$ having greater than mild PVL. ${ }^{23}$ In contrast, the transatrial approach can reduce the risk of LVOT obstruction by providing the surgeon with an opportunity to directly visualize the anterior leaflet and resect it if necessary, and in our experience has a decreased risk of procedural failure and reintervention. Although the anterior leaflet was resected in all 11 cases in this study, 2 patients had postoperative LVOT obstruction. Both had undergone TAVR before mitral valve surgery. We suspect that having 2 different stented valves protruding into the LVOT might have resulted in restricted blood flow, causing the obstructive effect. In patients with severe septal hypertrophy, septal myectomy or alcohol septal ablation before valve implantation should be considered because LVOT obstruction can still be present after excision of the anterior leaflet.

\section{Limitations}

This article has several limitations that should be addressed. The most substantial limitation is its small sample size, which makes meaningful statistical comparisons with other techniques difficult and underpowered. However, the ongoing Surgical Implantation of Transcatheter Valve in Native Mitral Annular Calcification trial will provide an opportunity to study this patient population in a larger series. ${ }^{18}$ This study also has an inherent selection bias because these patients were all considered high-risk surgical patients, which contributed to their selection for a transatrial approach. The short follow-up period limits any conclusions that can be made about long-term patient outcomes. All patients in this series underwent surgery by a single cardiac surgeon with extensive experience in transcatheter procedures, which limits the generalizability of the results to surgeons with adequate knowledge of and experience in utilizing transcatheter technology and may not apply to a nonexperienced facility or individual. 


\section{CONCLUSIONS}

Transatrial TMVR using a Sapien 3 THV for high-risk or prohibitive-surgical-risk patients with severe MAC can be performed safely with excellent short-term outcomes. To maximize the advantages of this technique, thorough preoperative assessment of anatomical features based on CT and echocardiogram, as well as careful patient selection, are necessary. Our experience indicates this technique can be applied to a currently underserved patient population that is not eligible for other surgical or transcatheter mitral valve interventions. Further studies with larger sample size are needed to evaluate the reproducibility and long-term efficacy of this technique.

\section{Conflict of Interest Statement}

Dr Kaneko is a speaker for Edwards Lifesciences, Abbott Laboratories, and Medtronic; and a consultant for Baylis and 4C Medical. All other authors reported no conflicts of interest.

The Journal policy requires editors and reviewers to disclose conflicts of interest and to decline handling or reviewing manuscripts for which they may have a conflict of interest. The editors and reviewers of this article have no conflicts of interest.

\section{References}

1. Carrel TP, Weber A. Selective, segmental decalcification: a safe alternative to extensive debridement of a severely calcified annulus during repair of mitral regurgitation. Interact Cardiovasc Thorac Surg. 2016;23:665-7.

2. Carpentier AF, Pellerin M, Fuzellier JF, Relland JY. Extensive calcification of the mitral valve anulus: pathology and surgical management. J Thorac Cardiovasc Surg. 1996;111:718-29.

3. Feindel CM, Tufail Z, David TE, Ivanov J, Armstrong S. Mitral valve surgery in patients with extensive calcification of the mitral annulus. J Thorac Cardiovasc Surg. 2003;126:777-82.

4. Said SM, Schaff HV. An alternate approach to valve replacement in patients with mitral stenosis and severely calcified annulus. J Thorac Cardiovasc Surg. 2014; 147:e76-8.

5. FDA expands indication for several transcatheter heart valves to patients at low risk for death or major complications associated with open-heart surgery [press release]. Available at: https://www.fda.gov/news-events/press-announcements/ fda-expands-indication-several-transcatheter-heart-valves-patients-low-risk-deathor-major. Accessed June 22, 2021

6. Himbert D, Bouleti C, Iung B, Nejjari M, Brochet E, Depoix JP, et al. Transcatheter valve replacement in patients with severe mitral valve disease and annular calcification. J Am Coll Cardiol. 2014;64:2557-8.

7. Guerrero M, Dvir D, Himbert D, Urena M, Eleid M, Wang DD, et al. Transcatheter mitral valve replacement in native mitral valve disease with severe mitral annular calcification: results from the first multicenter global registry. JACC Cardiovasc Interv. 2016;9:1361-71.
8. Guerrero M, Urena M, Himbert D, Wang DD, Eleid M, Kodali S, et al. 1-Year outcomes of transcatheter mitral valve replacement in patients with severe mitral annular calcification. J Am Coll Cardiol. 2018;71:1841-53.

9. Praz F, Khalique OK, Lee R, Veeragandham R, Russell H, Guerrero M, et al. Transatrial implantation of a transcatheter heart valve for severe mitral annular calcification. J Thorac Cardiovasc Surg. 2018;156:132-42.

10. Russell HM, Guerrero ME, Salinger MH, Manzuk MA, Pursnani AK, Wang D, et al. Open atrial transcatheter mitral valve replacement in patients with mitral annular calcification. J Am Coll Cardiol. 2018;72:1437-48.

11. El Sabbagh A, Eleid MF, Foley TA, Al-Hijji MA, Daly RC, Rihal CS, et al. Direct transatrial implantation of balloon-expandable valve for mitral stenosis with severe annular calcifications: early experience and lessons learned. Eur J Cardiothorac Surg. 2018:53:162-9.

12. Ghosh-Dastidar M, Bapat V. Transcatheter valve implantation in mitral annular calcification during open surgery: extended collar technique. Ann Thorac Surg. 2017; 104:e303-5.

13. Polomsky M, Koulogiannis KP, Kipperman RM, Cohen BM, Magovern CJ, Slater JP, et al. Mitral valve replacement with Sapien 3 transcatheter valve in severe mitral annular calcification. Ann Thorac Surg. 2017;103:e57-9.

14. Murashita T, Suri RM, Daly RC. Sapien XT transcatheter mitral valve replacement under direct vision in the setting of significant mitral annular calcification. Ann Thorac Surg. 2016;101:1171-4.

15. Abrams CLR, Weiner JP. Development and evaluation of the Johns Hopkins University risk adjustment models for Medicare+Choice plan payment. Final report to Centers for Medicare and Medicaid Services. Available at: https://www. hopkinsacg.org/document/development-and-evaluation-of-the-johns-hopkinsuniversity-risk-adjustment-models-for-medicarechoice-plan-payment/. Accessed June 22, 2021.

16. Murphy DJ, Ge Y, Don CW, Keraliya A, Aghayev A, Morgan R, et al. Use of cardiac computerized tomography to predict neo-left ventricular outflow tract obstruction before transcatheter mitral valve replacement. J Am Heart Assoc. 2017;6:e007353.

17. Stone GW, Adams DH, Abraham WT, Kappetein AP, Généreux P, Vranckx P, et al. Clinical trial design principles and end point definitions for transcatheter mitral valve repair and replacement: part 2: end point definitions: a consensus document from the Mitral Valve Academic Research Consortium. J Am Coll Cardiol. 2015;66:308-21.

18. Smith R. SITRAL trial and "transcatheter open" surgical approaches to patients with mac and $\mathrm{ms} / \mathrm{mr}$ - techniques and outcomes. Available at: https://www. tctmd.com/slide/sitral-trial-and-transcatheter-open-surgical-approaches-patientsmac-and-msmr-techniques-and. Accessed June 22, 2021.

19. Lamelas J, Alnajar A. Early outcomes for surgical minimally invasive Sapien 3 transcatheter mitral valve replacement. Ann Thorac Surg. November 2, 2020 [Epub ahead of print].

20. Kaneko T, Hirji S, Percy E, Aranki S, McGurk S, Body S, et al. Characterizing risks associated with mitral annular calcification in mitral valve replacement. Ann Thorac Surg. 2019;108:1761-7.

21. Külling M, Külling J, Wyss C, Hürlimann D, Reho I, Salzberg S, et al. Effective orifice area and hemodynamic performance of the transcatheter Edwards Sapien 3 prosthesis: short-term and 1-year follow-up. Eur Heart J Cardiovasc Imaging. 2018;19:23-30

22. Pibarot P, Dumesnil JG. Prosthetic heart valves: selection of the optimal prosthesis and long-term management. Circulation. 2009;119:1034-48.

23. Khan JM, Babaliaros VC, Greenbaum AB, Foerst JR, Yazdani S, McCabe JM, et al. Anterior leaflet laceration to prevent ventricular outflow tract obstruction during transcatheter mitral valve replacement. J Am Coll Cardiol. 2019;73: 2521-34.

Key Words: transatrial, transcatheter, mitral valve replacement, mitral annular calcification 